
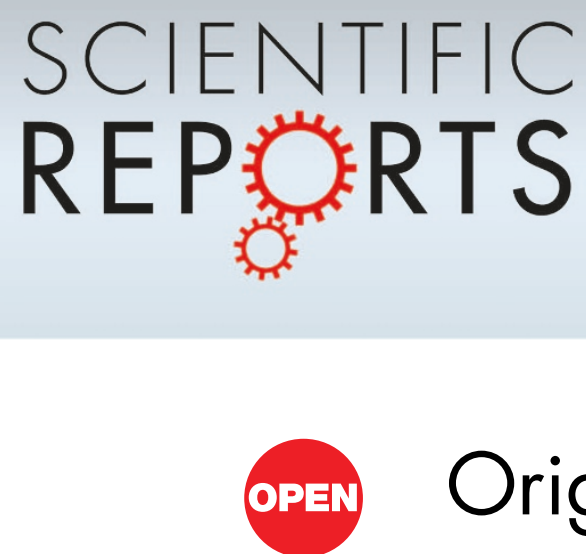

\title{
Origin of flaw-tolerance in nacre
}

\author{
Zaiwang Huang \& Xiaodong Li
}

\section{SUBJECT AREAS: \\ MECHANICAL \\ PROPERTIES \\ COMPOSITES \\ NANOPARTICLES}

STRUCTURAL PROPERTIES

Received

10 December 2012

Accepted

4 April 2013

Published

22 April 2013

Correspondence and requests for materials should be addressed to X.D.L. (lixiao@cec.sc.

edu)
Department of Mechanical Engineering, University of South Carolina, 300 Main Street, Columbia, South Carolina 29208, USA.

Over the past decades, our understanding of nacre's toughening origin has long stayed at the level of crack deflection along the biopolymer interface between aragonite platelets. It has been widely thought that the ceramic aragonite platelets in nacre invariably remain shielded from the propagating crack. Here we report an unexpected experimental observation that the propagating crack, surprisingly, invades the aragonite platelet following a zigzag crack propagation trajectory. The toughening origin of previously-thought brittle aragonite platelet is ascribed to its unique nanoparticle-architecture, which tunes crack propagation inside the aragonite platelet in an intergranular manner. For comparison, we also investigated the crack behavior in geologic aragonite mineral (pure monocrystal) and found that the crack propagates in a cleavage fashion, in sharp contrast with the intergranular cracking in the aragonite platelet of nacre. These two fundamentally different cracking mechanisms uncover a new toughening strategy in nacre's hierarchical flaw-tolerance design.

1 he nature of toughening mechanism in a material is, to a large extent, determined by how the crack tip interacts with material's inherent microstructure ${ }^{1-2}$. For instance, in coarse-grained metallic materials ${ }^{3}$, a wealth of lattice dislocation emission arising from the crack tip significantly relieves the stress concentration, consequently the imposed stress needs to be continuously increased to drive crack growth, leading to a considerable amount of energy-dissipation in fracture. Once the lattice dislocation activities are inhibited, a tendency to embrittlement will accordingly emerge. When the grain size of a coarse-grained metallic material is refined down to the nanometer-sized regime, the material's fracture toughness will be remarkably decreased due to the plastic deformation mechanism transition from the lattice dislocation slip to the grain boundary-mediated process ${ }^{4}$. Comparatively, monolithic ceramics are intrinsically stiff yet brittle ${ }^{5}$, their brittleness primarily suffers from scarce plastic deformation. As a result, the stress concentration can not be effectively released at the crack tip, resulting in poor energy-dissipation. Previous work ${ }^{6}$ has demonstrated that although decreasing the grain size of coarse-grained ceramics using the top-down fabrication technique can enhance their fracture toughness, this method typically involves a hypercritical non-equilibrium condition and unavoidably introduces an amount of impurities such as voids and contaminations into the nanocrystalline ceramics, posting shortcomings in investigating their inherent properties.

Nacre (mother-of-pearl) is widely renowned for its superior fracture toughness due to its exquisite structural hierarchy, despite of its primary structural component - brittle aragonite (a polymorph of $\left.\mathrm{CaCO}_{3}\right)^{7-11}$. It has been extensively demonstrated ${ }^{12-14}$ that nacre is a hierarchical nanocomposite, consisting of highly ordered polygonal aragonite platelets with the thickness of around 500 nanometers and the edge length of about $5 \mu \mathrm{m}$, sandwiched with 5-20 nanometer-thick organic biopolymer interlayer. Extensive work ${ }^{7-10,12-16}$ has shown that the toughening strategy in preventing crack catastrophic propagation in nacre arises from the staggered arrangement of aragonite platelets ${ }^{15}$, which regulates the crack path in a zigzag manner along the biopolymer interlayer between aragonite platelets. When a crack propagates within the biopolymer interlayer (with much lower stiffness) and encounters the ceramic-based aragonite platelet, the sharp discrepancy of mechanical stiffness can compel the crack deflected to its unfavorable heading ${ }^{15}$, i.e. crack growth direction relative to the imposed stress from perpendicular to parallel mode. Simultaneously, the deflection process additionally involves the resistance from the splitting of the atomic bonds between organic biomolecule and aragonite crystallite ${ }^{9}$, as well as the sliding resistance against the pull-out of aragonite platelets. Apparently, a substantial amount of energy-dissipation can be gained in the course of crack deflection, unlike the sharp crack cleavage in monolithic ceramics.

Recently, the finding ${ }^{17,18}$ of nanoparticles in individual aragonite platelets in nacre has attracted considerable attention. These nanoparticles are basic building blocks in nacre and assembled into individual pseudo-singlecrystal aragonite platelets, which jointly scatter into spot arrays in the electron diffraction pattern ${ }^{19,20}$. The nanoparticle-architecture is ascribed to play an important role in nacre's toughness amplification in conjunction with the "brick-mortar" microarchitecture ${ }^{17,21,22}$. However, those explorations lack of a quantitative data of how the nanoparticle-architecture plays the role in resisting the invading crack within individual aragonite platelets. 
The previous $\mathrm{s}^{23-25}$ nanomechanical characterization of individual aragonite platelets by nanoindentation focused on the measurement of elastic modulus and hardness. The crack propagation trajectory and its underlying physics of nanoparticle-architecture for defeating the crack remain completely unknown. Here we demonstrate direct experimental evidence that the crack propagates into an individual aragonite platelet in an intergranular manner, which has never been observed before. Equivalently important, this zigzag crack behavior is in sharp contrast with the catastrophic crack propagation in the geologic aragonite mineral (pure single-crystal). Our findings demonstrate that in addition to the crack deflection along the biopolymer interface between aragonite platelets, the nanoparticle-architecture within individual aragonite platelets offers additional crack extension resistance for nacre's toughness amplication. In the following section, we elaborate our findings on nacre's toughening mechanism and hierarchical flaw-tolerance design.

\section{Results}

Figure 1 reveals the interaction between crack and aragonite platelet, the primary structural component in nacre, and the crack path upon three-point bending loaded towards [002]. The experimental scheme and sample geometry are schematically depicted in Fig. 1a. After failure, the crack path, at a glance, indeed follows a zigzag profile (Fig. 1b) along the biopolymer interlayer, confirming the previously well-established theory that crack deflection is a critical energy-dissipation mechanism for nacre's toughness enhancement. However, a close-up examination, unexpectedly, disclosures a new cracking mechanism that crack can directly invade individual aragonite platelets. Let us select two typical areas (blue arrowed and red arrowed in
Fig. 1b) for demonstration, Fig. 1c displays the crack morphology in a nearly straight manner crossing two neighboring aragonite platelets (blue arrowed), suggesting that at least one of them has been invaded by the advancing crack taking into account the geometrically staggered arrangement of aragonite platelets in nacre. Meanwhile, it is particularly intriguing to note that crack propagation, after invasion, is not followed by successive cleavage of aragonite platelets, but subsequently deflected into biopolymer interlayer (black arrowed), implying that intra-platelet crack does not evolve into unstable state. Equivalently important, Fig. 1d shows the zigzag crack profile within individual aragonite platelets, indicative of the existence of inherent resistance to crack extension. In view of the previous understanding that individual aragonite platelets have been long regarded as brittle monocrystal ceramics and typically fail by cleavage along specific crystallographic plane, the finding that crack propagates in a zigzag manner is strikingly significant and how the crack is tuned in such a way needs further elaboration. Here we employed atomic force microscopy (AFM) to demonstrate more crack/aragonite platelet interaction details inside individual aragonite platelets. Fig. 1e reveals, for the first time, that the zigzag crack path is essentially along the boundaries of aragonite nanoparticles in an intergranular fashion. A closer examination (Fig. 1f) on the white boxed area in Fig. 1e clearly exposes the aragonite nanoparticles, and simultaneously, exhibits a bumpy edge in the form of particle boundary. We suggest that such zigzag crack behavior inside individual aragonite platelets resulted from nacre's unique nanoparticle-architecture.

To validate what we have observed - the zigzag crack behavior within individual aragonite platelets, Vickers indentation, with the stress condition different from three-point bending, was employed to
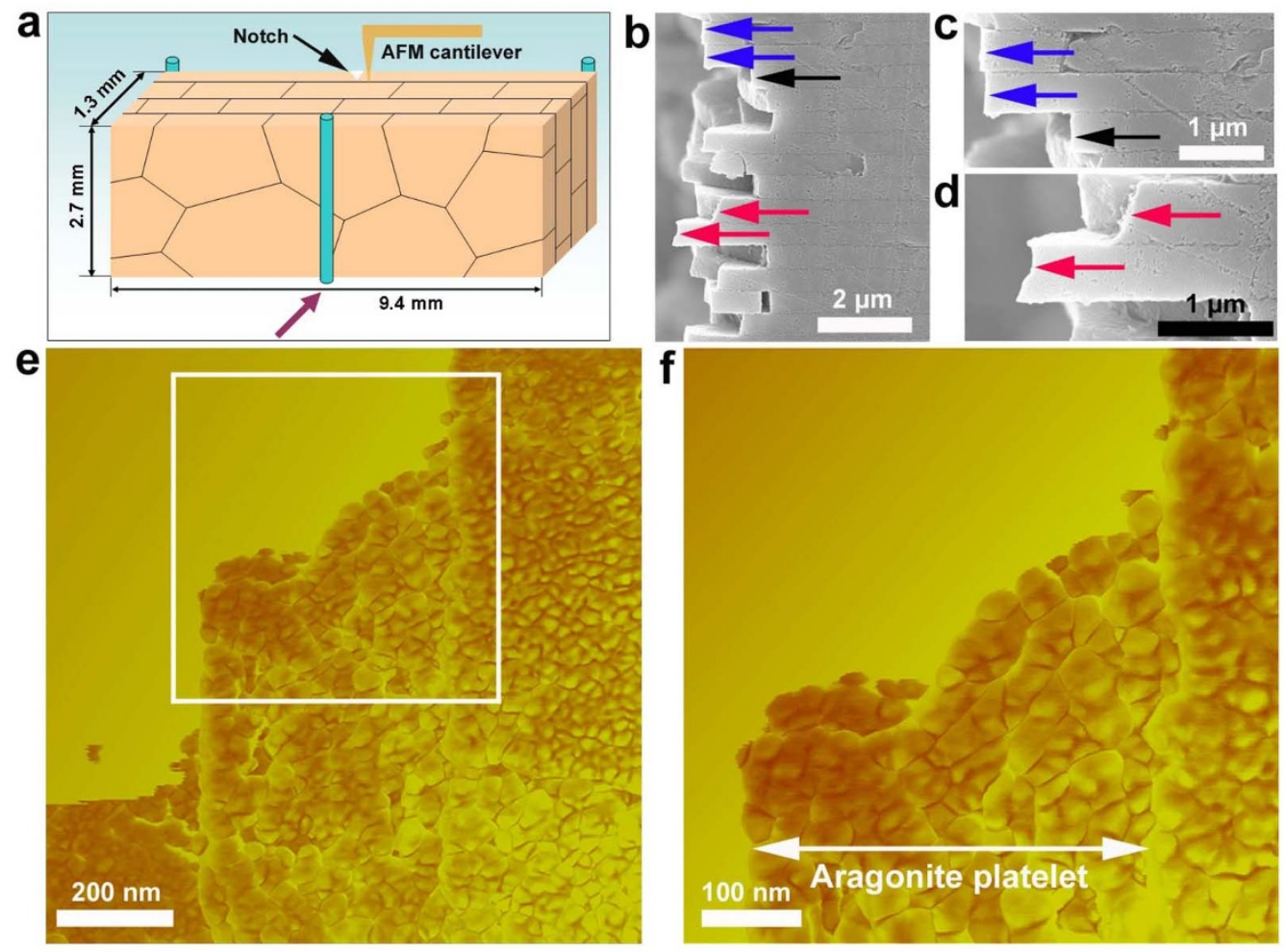

Figure $1 \mid$ Crack path profile and fractography within individual aragonite platelets. (a), Schematic drawing showing sample geometry and experimental scheme under three-point bending loaded along [002]. (b), Fracture path observation on nacre cross section, at a glance, indeed demonstrates crack deflection along its biopolymer interlayer in a zigzag manner. (c), A close-up view reveals at least one of two aragonite platelets (blue arrowed) has been invaded by an advancing crack considering geometrically staggered arrangement. Subsequently, however, the advancing crack is not followed by successive cleavage, but defected to biopolymer interlayer (black arrow). (d), Zigzag crack path shows the inherent extension resistance arising from the interior of individual aragonite platelets (red arrowed). (e), AFM observation confirms the zigzag crack profile within an individual aragonite platelet. (f), A closer examination (from white boxed area in Fig. 1e) reveals that crack propagation path is along the boundaries of aragonite nanoparticles. 
induce crack inside an aragonite platelet. Figure 2 demonstrates how the crack invades and interacts with the aragonite platelet. At the corner of indentation mark, AFM observation reveals that a zigzag crack (Fig. 2a) propagated and terminated inside the aragonite platelet, which is consistent with the crack morphology observed in Fig. If (Section 3, Supplementary materials). In order to uncover crystallographic details, an electron-transparent TEM thin foil containing the crack within the aragonite platelet was fabricated using focused ion beam (FIB) technique for lattice observation. The selected crack (white arrowed in Fig. 2b) from top-view along [002] can be found at the rightmost layer, adjacent to the platinum layer deposited by FIB. Fig. $2 c$ is the dark-field TEM observation showing that crack indeed traveled in a zigzag manner and the fracture circumference exposes a jagged edge. Careful geometrical measurement (Section 4, Supplementary materials) shows that the spacing and amplitude of the asperities of the jagged edge are on the order of a few nanometers, indicating that the crack was tuned by the inherent microstructure with the characteristic length scale of a few nanometers. In fact, this length scale is perfectly equivalent to the average particle size of aragonite nanoparticles ${ }^{19,26}$. In contrast to the considerable dislocation activities around the crack tip in coarse-grained metallic materials, no obvious lattice defects (Fig. 2d) such as dislocation and particle boundary sliding were detected in the vicinity of the intergranular crack, suggesting that the crack growth was neither controlled by direct atomic cleavage (straight), nor by dislocationrelated process. The origin of why the crack favors intergranular propagation can be attributed to its unique architecture, where highly-ordered aragonite nanoparticles ${ }^{19}$ together with their interparticle boundaries could be regarded as a nanocomposite that imposes an periodic structural effect ${ }^{27,28}$ on the stress status of crack tip. Although the structural chemistry of grain boundary still remains hotly debated, i.e. lattice dislocation ${ }^{19}$, amorphous aggregation ${ }^{18,19}$, these interfacial constituents are mechanically weak comparing with the aragonite particle interior and prone to suffer from stress damage. When a crack invades the aragonite platelet and encounters aragonite nanoparticles, it spontaneously chooses the weak grain boundary with less resistance as its propagation path. Wherein, the elaborate structural design with a characteristic length scale of a few nanometers offers periodic modulation of stress status at the crack tip, leading to a zigzag propagation path.

To fundamentally uncover that such an intergranular crack mechanism is unique for nacre's aragonite platelets, for comparison a naturally occurring geologic single-crystal aragonite was utilized to investigate its crack behavior. The geologic single-crystal aragonite sample with a pseudo-hexagonal profile (Fig. 3a) was identified to be of aragonite chemistry with single-crystal X-ray diffraction pattern (Section 2, Supplementary materials). On the top (002) plane, cracks propagated in a cleavage fashion from the corners of Vickers indentation mark (Fig. 3b), in sharp contrast with those in the nanoparticle-based aragonite platelets in nacre (Fig. 2a). The catastrophic crack cleavage in the geologic single-crystal aragonite is ascribed to the absence of inherent microstructure resistance, i.e. grain boundary

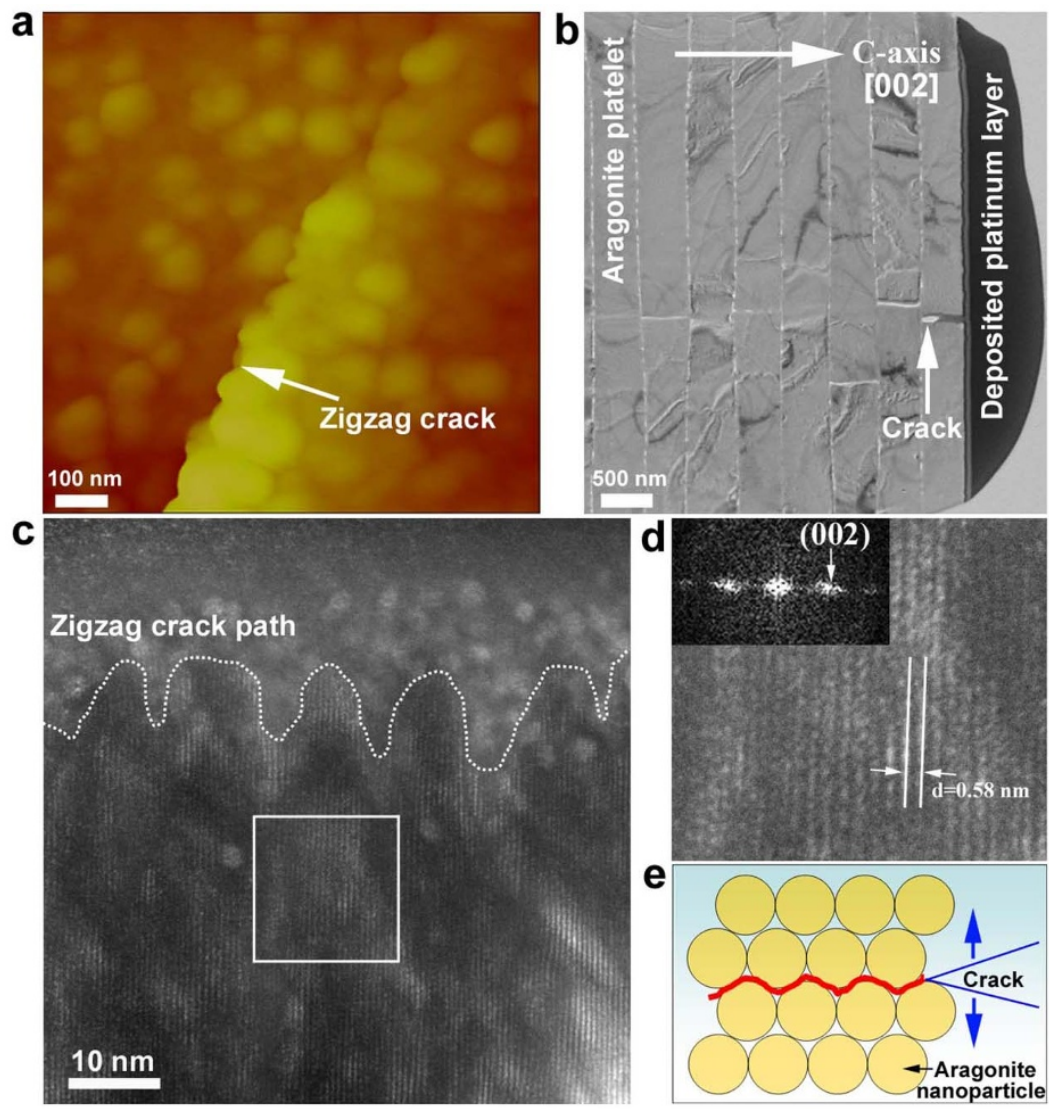

Figure $2 \mid$ Zigzag crack propagation path inside an individual aragonite platelet of nacre. (a), After imposing Vickers indentation on an aragonite platelet along [002] (Section 1, Supplementary materials), a typical crack radiated from the indentation mark corner was selected from AFM observation, showing the crack propagation path in a zigzag fashion. (b), An electron-transparent TEM foil containing one crack (white arrowed) was prepared by the FIB lift-out technique. (c), Dark-field TEM image showing wavy crack propagation path, the waviness is on the order of a few nanometers, in contrast with the interface geometry between aragonite platelet and biopolymer (Section 4, Supplementary materials). (d), A typical area near the fracture edge was selected for closer examination, showing that no discernible lattice defects such as dislocation and grain boundary sliding can be detected, as confirmed by single-crystal-like diffraction pattern using fast Fourier transform (FFT) measurement (inset). (e), Schematic illustration portraits that an advancing crack prefers the boundaries of aragonite nanoparticles as the propagation path with the least resistance. 

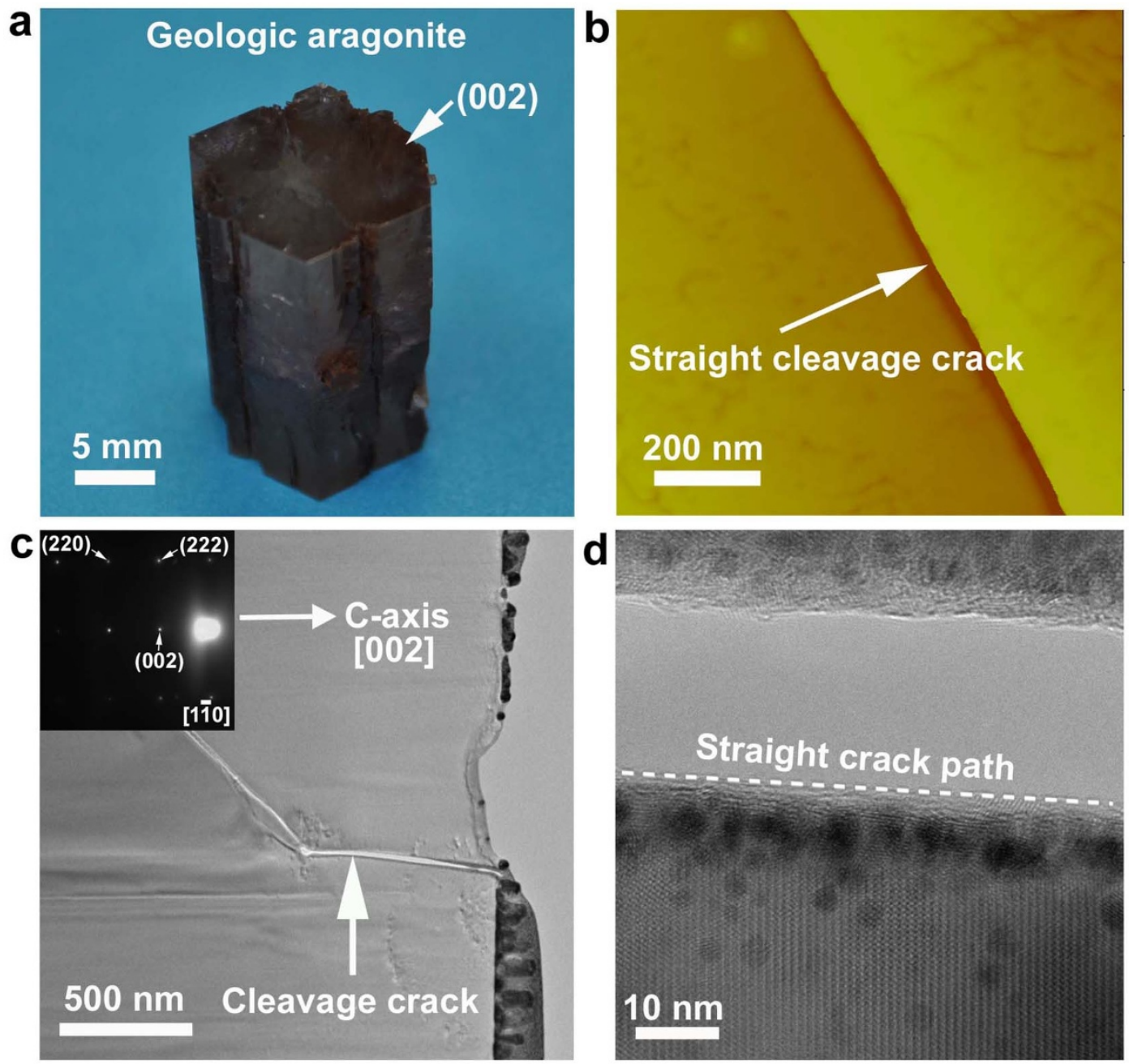

Figure 3 Crack behavior in geologic aragonite monocrystal. (a), The growth direction of geologic aragonite monocrystal with a pseudo-hexagonal profile on the top surface is toward [002]. (b), AFM observation shows that a representative crack, radiating from the Vickers indentation mark corner on crystallographic plane (002), cleaves aragonite crystal in a straight manner, indicative of brittle fracture mode. (c), A bright-field TEM image reveals crack invasion into the geologic aragonite monocrystal in a cleavage manner, the region around the crack was determined to be single-crystal by diffraction pattern (inset). (d), At the fracture edge, a closer view of lattice fringe exhibits straight crack cleavage behavior, in stark contrast with the intergranular manner within nacre's aragonite platelet. It is worthy pointing out that the "dark" dots around the fracture edge were caused by the unavoidable contamination during the FIB fabrication, i.e. platinum implantation.

and second-phase. To further demonstrate the lattice activity details along the crack pathway, TEM thin foil containing an advancing crack was prepared by FIB technique. Fig. $3 \mathrm{c}$ is the bright-field TEM observation of the crack path in the geologic single-crystal aragonite. A close-up view of the crack (white arrowed) reveals a typical brittle fracture in the form of straight crack path with an atomic level smooth edge, in sharp contrast with the periodic bumpy edge observed on the crack path of nacre's aragonite platelets. (Fig. 3d).

\section{Discussion}

A cube-corner nanoindenter was used to probe the mechanical response difference between the geologic single-crystal aragonite and nacre's aragonite platelets. Figure 4 depicts the load-displacement curves of nanoindentations made on the (002) planes of the respective geologic single-crystal aragonite and nacre's aragonite platelet with the peak indentation load $(300 \mu \mathrm{N})$ (Section 5, Supplementary materials). For the geologic single-crystal aragonite, at least two pronounced displacement pop-ins (strain avalanche) can be detected in the loading curve and the first one occurs at the displacement of approximately $6.6 \mathrm{~nm}$. In this aspect, the indentation depth is sufficiently small and its corresponding contact zone can be considered as the nominally defect-free volume ${ }^{29,30}$. The sudden displacement excursion is a result of plasticity onset, immediately after perfect elasticity process. Triggering such plastic deformation requires the stress level to be on the order of theoretical strength $(\sim \mathrm{E} / 10)^{29,30}$. Obviously, the stress concentration at the first pop-in undoubtedly reached the theoretical strength, initiating the plastic deformation onset. In comparison, no discernible pop-in can be detected in the nanoindentation loading curve for nacre's individual aragonite platelets, implying the stress concentration underneath the nanoindenter did not achieve the theoretical strength. Gao et al. ${ }^{16}$ proposed that for a brittle material, there exists a critical length scale based on Griffith criterion as follows

$$
h^{*} \approx \alpha^{2} \frac{\gamma E_{m}}{\sigma_{t h}^{2}}
$$

where $h^{*}$ is the critical length scale, parameter $\alpha$ is equal to $\sqrt{\pi}, \gamma$ is the surface energy, $\mathrm{E}_{\mathrm{m}}$ and $\sigma_{\mathrm{th}}$ are elastic modulus and theoretical strength, respectively. For the brittle geologic monocrystal aragonite ${ }^{16}$, the critical length scale $h^{*}$ is estimated to be approximately 30 nanometers. When the aragonite particle size is below or equival- 


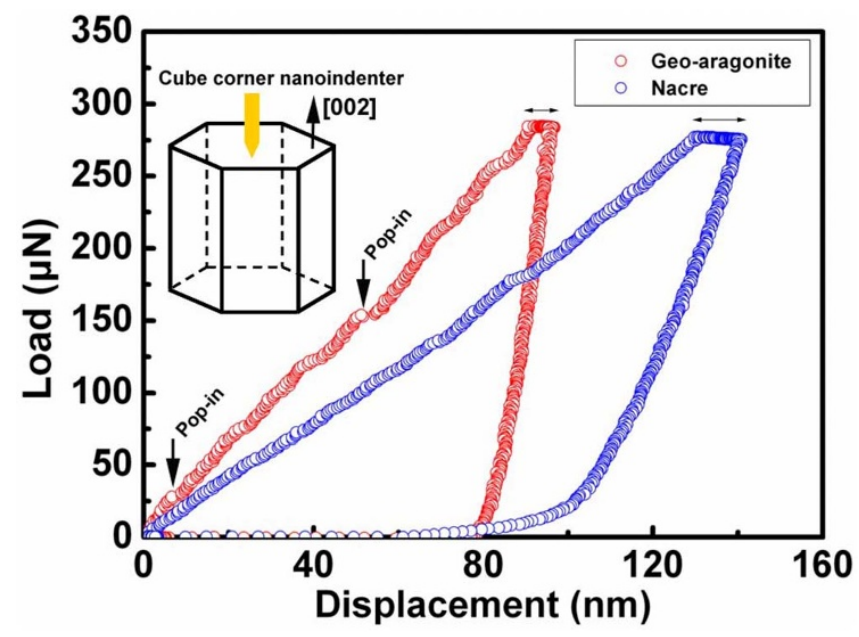

Figure $4 \mid$ Nanoindentation load-displacement response from nacre's aragonite platelets and geologic aragonite monocrystal along [002]. For the geologic aragonite monocrystal, at the nanoindentation displacement of approximately 6.6 nanometers, a sudden displacement pop-in was found, which is consistent with the plasticity onset at the stress level of the theoretical strength. In comparison, no discernible pop-in event can be detected for nacre's aragonite platelets, implying the stress underneath the nanoindenter was not accumulated to the theoretical strength. Its lower loading slope and more apparent viscoelastic effect at the peak indentation holding segment jointly indicate the existence of biopolymer matrix, buffering stress concentration within individual aragonite platelets.

ent to this critical length scale, the stress concentration can not be accumulated anymore, as reflected by the smooth loading curve for nacre's aragonite platelets. Prior to achieving the theoretical strength, the weak nanoparticle boundary can serve as the relieving outlet for potential stress accumulation. Whereas, once the grain size far exceeds the critical length scale, i.e. the geologic monocrystal aragonite, the stress upon deformation can be significantly concentrated, as validated by the onset of first pop-in event under nanoindentation. Here, we can reasonably argue when the aragonite particle size falls into coarse-grained regime (micrometer-scale), the stress concentration upon deformation accordingly emerges and triggers catastrophic brittle fracture (Section 6, Supplementary materials).

The association between length scale and deformation mechanism, i.e. dislocation in metallic material, shear band in metallic glass, crack in ceramic, is a critically important guideline of how to acquire fracture toughness from microstructure design. The fracture toughness of metallic materials strongly depends on the spacing between dislocation nucleation sites and obstacles ${ }^{3}$. Owing to its micrometer-sized affected volume surrounding crack tip, the attempt to keep such a small length scale via refining grain size, will hence impose potential microstructure obstacles on dislocation activities at the crack tip. Likewise, the characteristic microstructure length scale associated to the maximum spatial extension for shear band has also been found in metallic glasses ${ }^{31,32}$. Through introducing crystalline imhomogeneities in metallic glasses, the traveling distance of shear band will be shortened prior to evolving into an unstable crack, thereby avoiding catastrophic failure. Similarly, it is conceivable that for ceramic materials tailoring the length scale such as embedding second-phase in matrix to intercept crack propagation will enhance the crack extension resistance, which will in turn contributes to fracture toughness improvement. However, this toughening approach typically suffers from stress concentration at the crack tip prior to being intercepted. Whereas, the finding of crack manifestation in individual aragonite platelets works out a new critical length scale, referring to a criterion whether there exists stress concentration at the crack tip.
Of course, the role of nanoparticle boundary in biominerals, relative to other pure nanocrystalline ceramics, is probably unique in releasing the stress concentration ${ }^{33}$. In this regard, its exceptional role can be validated by two characteristic traits from the load-displacement response. Through comparing with geologic monocrystal aragonite, the load-displacement curve for nacre's aragonite platelets exhibits a much lower loading slope, and more remarkable viscoelastic effect in the nanoindentation holding segment, implying the existence of biopolymer matrix (soft phase) inside nacre's individual aragonite platelets. It is worthy pointing out that several slight fluctuations in the loading curve of nacre's aragonite platelets can not be regarded as displacement pop-ins, but related to the deformation response from aragonite nanoparticle rotation (Section 7, Supplementary materials).

The direct evidence that individual aragonite platelets defeat crack catastrophic propagation with its nanoparticle-architecture overturns the previous understanding that considers nacre's aragonite platelet as simply brittle monocrystal. Such a cracking mechanism favors local buffering in case a crack rigidly invades the aragonite platelet. Certainly, it is not expected for aragonite platelets to witness a catastrophic failure arising from confined stress concentration. This manifestation is reminiscent to the mechanical protection role of individual threads of spider webs, where local damage-control promotes the integral mechanical optimization ${ }^{34,35}$. The nanoscale localized damage-tolerance design principle in nacre is probably ubiquitous for other biominerals, i.e. tooth $^{36}$, bone $\mathrm{e}^{37}$, sea urchin spine $^{38}$, ancient armored fish ${ }^{39}$.

\section{Methods}

In this letter, natural nacre materials from California red abalone (Haliotis rufescens) that belong to the class of gastropoda were studied. The shells were collected alive in Santa Barbara, CA. Nacre samples were cut from the nacreous layer of the shell with a water-cooled, low-speed diamond saw. The three-point bending specimen with a notch scratched using a razor blade was tested along $\mathrm{C}$-axis direction, and failure path on the cross section was subsequently used for AFM observation (Veeco Dimension 3100, Veeco Group), and scanning electron microscopy observation (Zeiss Ultra Thermal Field Emission). To enable that the nanoindentation depth under the cube corner nanoindenter (Triboscope nanomechancial testing system, Hysitron Inc.) is restrictively limited within one individual aragonite platelet, a simple method using a razor blade (Section 1, Supplementary materials) to peel off the nacre sample along cross section was used to create a freshly cleavage surface (aragonite platelet plane) for the subsequent nanoindentation and Vickers indentation experiments. In addition, we employed the naturally occurring geologic aragonite monocrystal as a reference for comparison to probe the corresponding nanoindentation and Vickers indentation behaviors. The geologic aragonite monocrystal was identified as single crystal by Xray tomography (Section 2, Supplementary materials). At the Vickers indenter corners, cracks in the respective nacre's aragonite platelet and geologic aragonite monocrystal were located and the corresponding transmission electron microscopy (TEM) thin foils were fabricated using focused ion beam (FIB) technique (FEI Quanta 3D FEG). The TEM samples were observed using Hitachi HF2000 with an accelerating voltage of $200 \mathrm{KV}$.

1. Kumar, S. \& Curtin, W. A. Crack interaction with microstructure. Mater. Today 10, 34-44 (2007).

2. Ritchie, R. O. The conflicts between strength and toughness. Nat. Mater. 10, 817-822 (2011).

3. Chakravarthy, S. S. \& Curtin, W. A. Origin of Plasticity Length-Scale Effects in Fracture. Phys. Rev. Lett. 105, 115502 (2010).

4. Hasnaoui, A., Van Swygenhoven, H. \& Derlet, P. M. Dimples on nanocrystalline fracture surfaces as evidence for shear plane formation. Science 300, 1550-1552 (2003).

5. Lawn, B. R., Padture, N. P., Cai, H. D. \& Guiberteau, F. Making ceramics ductile. Science 263, 1114-1116 (1994).

6. Ovid'ko, I. A. \& Sheinerman, A. G. Micromechanisms for improved fracture toughness in nanoceramics. Rev. Adv. Mater. Sci. 29, 105-125 (2011).

7. Currey, J. D. Mechanical-properties of mother of pearl in tension. Proc. R. Soc. Lond. B 196, 443-463 (1977).

8. Jackson, A. P., Vincent, J. F. V. \& Turner, R. M. The mechanical design of nacre. Proc. R. Soc. Lond. B 234, 415-440 (1988).

9. Smith, B. L. et al. Molecular mechanistic origin of the toughness of natural adhesives, fibres and composites. Nature 399, 761-763 (1999).

10. Kamat, S., Su, X., Ballarini, R. \& Heuer, A. H. Structural basis for the fracture toughness of the shell of the conch Strombus gigas. Nature 405, 1036-1040 (2000). 
11. Ortiz, C. \& Boyce, M. C. Materials science - Bioinspired structural materials. Science 319, 1053-1054 (2008).

12. Meyers, M. A., Chen, P.-Y., Lin, A. Y.-M. \& Seki, Y. Biological materials: Structure and mechanical properties. Prog. Mater. Sci. 53, 1-206 (2008).

13. Espinosa, H. D., Rim, J. E., Barthelat, F. \& Buehler, M. J. Merger of structure and material in nacre and bone - Perspectives on de novo biomimetic materials. Prog. Mater. Sci. 54, 1059-1100 (2009).

14. Wang, R. \& Gupta, H. S. Deformation and Fracture Mechanisms of Bone and Nacre. Annu. Rev. Mater. Res. 41, 41-73 (2011).

15. Wang, R. Z., Wen, H. B., Cui, F. Z., Zhang, H. B. \& Li, H. D. Observations of damage morphologies in nacre during deformation and fracture. J. Mater. Sci. 30, 2299-2304 (1995).

16. Gao, H. J., Ji, B. H., Jager, I. L., Arzt, E. \& Fratzl, P. Materials become insensitive to flaws at nanoscale: Lessons from nature. Proc. Natl. Acad. Sci. USA 100, 5597-5600 (2003).

17. Li, X. D., Chang, W. C., Chao, Y. J., Wang, R. Z. \& Chang, M. Nanoscale structural and mechanical characterization of a natural nanocomposite material: The shell of red abalone. Nano Lett. 4, 613-617 (2004).

18. Rousseau, M. et al. Multiscale structure of sheet nacre. Biomaterials 26, 6254-6262 (2005)

19. Li, X. D. \& Huang, Z. W. Unveiling the Formation Mechanism of Pseudo-SingleCrystal Aragonite Platelets in Nacre. Phys. Rev. Lett. 102, 075502 (2009).

20. Huang, Z. \& Li, X. Order-disorder transition of aragonite nanoparticles in nacre. Phys. Rev. Lett. 109, 025501 (2012).

21. Li, X. D., Xu, Z.-H. \& Wang, R. Z. In situ observation of nanograin rotation and deformation in nacre. Nano Lett. 6, 2301-2304 (2006).

22. Sun, J. Y. \& Bhushan, B. Hierarchical structure and mechanical properties of nacre: a review. Rsc Advances 2, 7617-7632 (2012).

23. Bruet, B. J. F. et al. Nanoscale morphology and indentation of individual nacre tablets from the gastropod mollusc Trochus niloticus. J. Mater. Res. 20, 2400-2419 (2005).

24. Barthelat, F., Li, C.-M., Comi, C. \& Espinosa, H. D. Mechanical properties of nacre constituents and their impact on mechanical performance. J. Mater. Res. 21, 1977-1986 (2006).

25. Katti, K. S., Mohanty, B. \& Katti, D. R. Nanomechanical properties of nacre. J. Mater. Res. 21, 1237-1242 (2006).

26. Huang, Z. W. et al. Uncovering high-strain rate protection mechanism in nacre Sci. Rep. 1, 148 (2011).

27. Fratzl, P., Gupta, H. S., Fischer, F. D. \& Kolednik, O. Hindered crack propagation in materials with periodically varying Young's modulus - Lessons from biological materials. Adv. Mater. 19, 2657-2661 (2007).

28. Kolednik, O., Predan, J., Fischer, F. D. \& Fratzl, P. Bioinspired Design Criteria for Damage-Resistant Materials with Periodically Varying Microstructure. Adv. Funct. Mater. 21, 3634-3641 (2011).

29. Bei, H., Lu, Z. P. \& George, E. P. Theoretical strength and the onset of plasticity in bulk metallic glasses investigated by nanoindentation with a spherical indenter. Phys. Rev. Lett. 93, 125504 (2004).
30. Bei, H., George, E. P., Hay, J. L. \& Pharr, G. M. Influence of indenter tip geometry on elastic deformation during nanoindentation. Phys. Rev. Lett. 95, 045501 (2005).

31. Hofmann, D. C. et al. Designing metallic glass matrix composites with high toughness and tensile ductility. Nature 451, 1085-1089 (2008).

32. Demetriou, M. D. et al. A damage-tolerant glass. Nat. Mater. 10, 123-128 (2011).

33. Kim, Y.-Y. et al. An artificial biomineral formed by incorporation of copolymer micelles in calcite crystals. Nat. Mater. 10, 890-896 (2011).

34. Cranford, S. W., Tarakanova, A., Pugno, N. M. \& Buehler, M. J. Nonlinear material behaviour of spider silk yields robust webs. Nature 482, 72-78 (2012).

35. Omenetto, F. G. \& Kaplan, D. L. Spider webs: Damage control. Nat. Mater. 11, 273-274 (2012)

36. Imbeni, V., Kruzic, J. J., Marshall, G. W., Marshall, S. J. \& Ritchie, R. O. The dentin-enamel junction and the fracture of human teeth. Nat. Mater. 4, 229-232 (2005).

37. Tai, K., Dao, M., Suresh, S., Palazoglu, A. \& Ortiz, C. Nanoscale heterogeneity promotes energy dissipation in bone. Nat. Mater. 6, 454-462 (2007).

38. Seto, J. et al. Structure-property relationships of a biological mesocrystal in the adult sea urchin spine. Proc. Natl. Acad. Sci. USA 109, 3699-3704 (2012).

39. Bruet, B. J. F., Song, J., Boyce, M. C. \& Ortiz, C. Materials design principles of ancient fish armour. Nat. Mater. 7, 748-756 (2008).

\section{Acknowledgements}

We thank the help from K. Wong and R. Garcia for FIB preparation and TEM observation, respectively, at North Carolina State University's Analytical Instrumentation Facility (AIF). We also appreciate the help with SEM observation from Y. C. Yang (EM Center, University of South Carolina). This work was supported by the U. S. Army research office under agreement/grant No. W911 NF-07-1-0449.

\section{Author contributions}

Z. H. and X. L. designed the project. Z. H. performed the experiments. Z. H. and X. L. wrote this paper.

\section{Additional information}

Supplementary information accompanies this paper at http://www.nature.com/ scientificreports

Competing financial interests: The authors declare no competing financial interests.

License: This work is licensed under a Creative Commons

Attribution-NonCommercial-NoDerivs 3.0 Unported License. To view a copy of this license, visit http://creativecommons.org/licenses/by-nc-nd/3.0/

How to cite this article: Huang, Z.W. \& Li, X.D. Origin of flaw-tolerance in nacre. Sci. Rep. 3, 1693; DOI:10.1038/srep01693 (2013) 